\title{
Two-dimensional semantics and the nesting problem*
}

\author{
David J. Chalmers and Brian Rabern
}

July 2, 2013

\section{Introduction}

Graeme Forbes (2011) raises some problems for two-dimensional semantic theories. The problems concern nested environments: linguistic environments where sentences are nested under both modal and epistemic operators. Closely related problems involving nested environments have been raised by Scott Soames (2005) and Josh Dever (2007). Soames (forthcoming) goes so far as to say that nested environments pose the "chief technical problem" for strong two-dimensionalism. We might call the problem of handling nested environments within two-dimensional semantics the nesting problem.

We concentrate especially on Forbes's version of the nesting problem here, with some attention to Dever's version as well (Chalmers 2011a discusses Soames). Forbes concentrates especially on sentences in which a factive epistemic operator, such as 'Someone establishes that' or 'It is a priori that' is embedded within a further modal or epistemic operator. His key examples are versions of the following: ${ }^{1}$

(1) Necessarily, if someone establishes that Hesperus has no moon, then Hesperus has no moon.

(2) It is a priori that if someone establishes that Hesperus is Phosphorus, then Hesperus is Phosphorus.

(3) Necessarily, if it is a priori that Julius invented the zip, then Julius invented the zip.

Intuitively, (1)-(3) are all true. Let us say that an operator $O$ is materially factive when $O \phi \supset \phi$ is true for all sentences $\phi$, that $O$ is modally factive when $\square(O \phi \supset \phi)$ is true for all $\phi$, and that $O$ is epistemically factive when $\mathbf{\square}(O \phi \supset \phi)$ is true for all $\phi$. (We use ' $\square$ ' for the 'Necessarily' operator and ' $\boldsymbol{\square}$ ' for the 'It is a priori that' operator throughout). ${ }^{2}$ Then (1)

\footnotetext{
*Thanks to Peter Fritz.

${ }^{1}(1),(2)$, and (3) are versions of Forbes' sentences (3), (5), and (6), with minor changes to the latter two for uniformity and simplicity, and with "a priori" in place of "epistemically necessary".

${ }^{2}$ Note that throughout the article we will often rely on the linguistic context to distinguish between the use and mention of symbols and formulae instead of cluttering the presentation with quotes and corner-quotes.
} 
and (2) are instances of the plausible claims that 'Someone establishes' is modally factive and epistemically factive respectively. (3) is an instance of the plausible claim that 'It is a priori that' is modally factive.

Forbes argues that two-dimensionalism has a hard time explaining the truth of (1)-(3). He says that simple versions of two-dimensionalism are refuted by cases such as (1), and that more complex versions (such as that developed in Chalmers 2011a) are threatened by cases such as (2) and (3). His basic objection is that given the way that two-dimensionalism treats epistemic operators, it is difficult for the view to accommodate their modal and epistemic factivity.

In fact, the two-dimensional semantics for attitude ascriptions developed in Chalmers (2011a) has no trouble accommodating the truth of (1) and (2). (3) raises an interesting puzzle, but this puzzle arises for anyone who accepts the contingent a priori, quite independently of two-dimensional semantics. The resources of two-dimensional semantics turn out to offer a promising resolution of the puzzle.

In what follows, we first lay out the basic principles of two-dimensional semantics and a simple treatment of necessity and apriority operators, and spell out how Forbes' puzzle (3) arises within this framework. We then show how a generalized version of the puzzle arises independently of two-dimensional semantics. We go on to spell out a two-dimensional treatment of attitude verbs and show how this handles Forbes' puzzles (1) and (2). Finally, we spell out a two-dimensional treatment of the apriority operator that fits the two-dimensional treatment of attitude verbs and show how it resolves Forbes' puzzle (3).

\section{Two-dimensional semantics}

In a two-dimensional semantics the extensions of expressions are relativized to pairs of possibilia. We will call the first element a scenario and the second element a world. Here worlds are metaphysically possible worlds. In epistemic two-dimensional semantics (Chalmers 2006), scenarios are epistemically possible situations: ways things might be that cannot be ruled out a priori. Scenarios are often modeled as centered metaphysically possible worlds, though other understandings are available. For present purposes these details will not matter and we will simply understand scenarios to be (uncentered) possible worlds.

The extension of an expression $\alpha$ at a scenario-world pair $(v, w)$ is denoted by $\llbracket \alpha \rrbracket^{v, w}$. (Extensions can be relativized to an interpretation and a variable assignment and also to a context, but for simplicity we will leave the former relativizations implicit and abstract away from the latter.) Extensions for sentences are truth-values; extensions for singular terms are individuals; extensions for predicates are properties (or classes); and so on. An expression's associated function from a scenario-world pair $(v, w)$ to an extension is its twodimensional intension (or two-dimensional matrix). We can say that mat $(\alpha)=\lambda v \lambda w \cdot \llbracket \alpha \rrbracket^{v, w}$ is the two-dimensional intension of an expression $\alpha$.

Given an expression $\alpha$ with a two-dimensional intension $F$, various aspects of this intension can be singled out as theoretically useful semantic values. The primary intension of $\alpha, \operatorname{pri}(\alpha)$, is $\lambda v \cdot \llbracket \alpha \rrbracket^{v, v}$. This is the diagonal of the two-dimensional intension: it maps 
every scenario $v$ to $F(v, v)$. The secondary intension of $\alpha$ relative to a scenario $v$, $\sec _{v}(\alpha)$, is $\lambda w \cdot \llbracket \alpha \rrbracket^{v, w}$. This is the "row" of the matrix corresponding to $v$ : it maps every world $w$ to $F(v, w)$. The secondary intension of $\alpha$ (simpliciter), $\sec (\alpha)$, is $\sec _{@}(\alpha)$. This is the "row" of the matrix corresponding to the actual world @: it maps every world $w$ to $F(@, w)$.

In the epistemic two-dimensional framework, primary and secondary intensions are closely connected to apriority and necessity respectively. For example, consider Evans' descriptive name 'Julius', introduced for whoever invented the zip (Evans 1979). The primary intension of 'Julius' picks out whoever invented the zip in a given scenario, while its secondary intension picks out the actual inventor (William C. Whitworth) in all worlds. Correspondingly, Evans' contingent a priori sentence 'Julius invented the zip (if anyone did)' has a primary intension that is true at all scenarios (reflecting its apriority), while it has a secondary intension that is false at some worlds (reflecting its contingency). ${ }^{3}$

Likewise, 'Hesperus' and 'Phosphorus' have the same secondary intension but different primary intensions. For a rigid designator, the secondary intension will pick out the actual extension in all worlds, so the secondary intensions of both 'Hesperus' and 'Phosphorus' pick out Venus in all worlds. The primary intension of most expressions is, at least to a first approximation, akin to a sort of associated descriptive content. So the primary intension of 'Hesperus' picks out (something like) the brightest object in the evening sky in a scenario, and the primary intension of 'Phosphorus' picks out something like the brightest object in the morning sky. Accordingly, the necessary a posteriori sentence 'Hesperus is Phosphorus' has a secondary intension that is true at all worlds (reflecting its necessity) and a primary intension that is false at some scenarios (reflecting its aposteriority).

Given the connection between secondary intensions and metaphysical modality and the connection between primary intensions and the a priori, it would be quite natural, in a twodimensional framework, to provide the following semantics for $\square$ (the metaphysical necessity operator) and $\mathbf{\square}$ (the apriority or epistemic necessity operator):

- $\llbracket \square \phi \rrbracket^{v, w}=1$ iff for all $w^{\prime} \llbracket \phi \rrbracket^{v, w^{\prime}}=1$

- $\llbracket \boldsymbol{\square} \phi \rrbracket^{v, w}=1$ iff for all $v^{\prime} \llbracket \phi \rrbracket^{v^{\prime}, v^{\prime}}=1$

That is, 'Necessarily $\phi$ ' is true at $(v, w)$ iff for all worlds $w$ ', the embedded sentence $\phi$ is true at $\left(v, w^{\prime}\right)$. And 'It is a priori that $\phi$ ' is true at $(v, w)$ iff for all scenarios $v^{\prime}$, the embedded sentence $\phi$ is true at $\left(v^{\prime}, v^{\prime}\right)$.

These basic details are enough to pose the nesting problem. Given the semantics above, a sentence such as (3) will come out false. If we let $\phi$ be a contingent a priori sentence such as 'Julius invented the zip', then the primary intension of $\phi$ will be true at all scenarios. It follows that the two-dimensional intension of $\phi$ is true at all pairs $(v, w)$, so that the secondary intension of $\phi$ is true at all worlds. But $\phi$ is contingent, so there is a world $w$ at which the secondary intension of $\phi$ is false. At $w$, the secondary intension of $\phi \supset \phi$ is false. So (3) is false.

\footnotetext{
${ }^{3}$ The connection between apriority and necessity of primary intension may be either substantive or stipulative depending on just how the key notions are defined. We abstract away from those issues here.
} 
The problem here arises because as defined, $\mathbf{\square}$ is a pure epistemic operator: that is, the two-dimensional intension of $\phi$ depends only on the primary intension of $\phi$, and not on the secondary intension or any other aspect of the two-dimensional intension of $\phi$. It is easy to see that if there are contingent a priori sentences, the apriority operator cannot be both a pure epistemic operator and modally factive. ${ }^{4}$

It follows that a two-dimensionalist must either deny that apriority is a pure epistemic operator or deny that it is modally factive. We think that both options are coherent. Regarding the second option: the pure epistemic apriority operator defined above is well-defined, but because it is not modally factive, it is arguably not the best candidate for what is expressed by 'It is a priori that' in philosophical English. ${ }^{5}$ So we will ultimately take the first option, giving a revised two-dimensional treatment of the apriority operator on which it is not a pure epistemic operator. First, however, we will show that a version of this puzzle is a puzzle for everyone.

\section{The generalized nesting problem}

The nesting problem for two-dimensional semantics is a special case of a general problem about the interaction of modal and epistemic operators that arises independently of twodimensional semantics. We can put the generalized nesting problem as follows.

The following three sentences are plainly inconsistent:

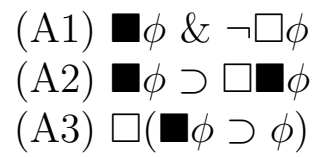

The inconsistency here arises for any two modal operators $\square$ and $\boldsymbol{\square}$, requiring only that satisfies the distribution axiom $\square(\chi \supset \psi) \supset(\square \chi \supset \square \psi){ }^{6}{ }^{6}$ When we understand the two operators as necessity and a priority, (A1) says that $\phi$ is contingent and a priori. (A2) is an instance of the claim that apriority entails necessary apriority. (A3) is an instance of the claim that apriority is modally factive.

All of these claims are initially plausible, independently of two-dimensional semantics. If

\footnotetext{
${ }^{4}$ This problem is a version of Dever's "nesting argument" (2007). Dever finds a version of the argument in Soames (2005) pp. 278-279, and Forbes finds a version of the argument (construed as an argument against Fregean theories) in Richard (1993) pp. 246-251, though the details of each are quite different. Chalmers (2011a, footnote 24) gives a brief reply.

${ }^{5}$ There are various apriori-like notions that are not modally factive. For example, Davies and Humberstone's (1980) operator fixedly actually and an operator corresponding to Kaplan's (1989) notion of indexical validity (truth at every context) are not modally factive. See also Fritz (forthcoming), who favors a twodimensional logic in which apriority is not modally factive.

${ }^{6}$ This inconsistent triad is at the core of "version 2" of the nesting argument in Dever 2007 (p. 11). The form is somewhat obscured by Dever's invoking additional ancillary assumptions and by his use of two propositions $\phi$ and $\psi$ rather than one ( $\phi$ here corresponds to $\phi \leftrightarrow \psi$ there), but it is clear on examination that his contradiction is generated by versions of the three principles above.
} 
there are contingent a priori sentences, then we can choose $\phi$ to be one of those sentences and there is no question about (A1). Furthermore, if $\boldsymbol{\nabla} \phi$ is understood in the standard way as $\diamond K^{A}(\phi)$, where $K^{A}$ is the operator 'someone knows a priori that', then (A2) is a consequence of modal axiom S5. (A3) is the intuitive claim of modal factivity that Forbes appeals to in his argument. Nevertheless, one of the three must go.

The problem here is closely connected to the nesting problem in the previous section. That problem was in effect generated by (A1), (A3), and the claim that apriority is a pure epistemic operator. The latter claim is not the same as (A2), but it leads to (A2) given weak assumptions. So in effect, all that two-dimensional semantics adds to the generalized nesting problem is an additional consideration in favor of (A2), on top of the consideration in the previous paragraph.

Indeed, the generalized problem clearly arises on common Russellian views of propositions (e.g. Soames 2007). On such a view, when $\phi$ expresses a contingency, ' $\phi$ iff actually $\phi$ ' expresses a Russellian proposition $p$ that is contingent and knowable a priori. Consider a case in which $p$ is actually known a priori. Then there will be worlds $w$ in which $p$ is false (as $\phi$ is false in $w$ and true in the actual world) but relative to which it is possible that someone know $p$ a priori (as $p$ is known a priori in the actual world, which is possible relative to $w$ ). So a priori knowability is not modally factive. It may even be that there are propositions that are known a priori in counterfactual worlds while being false in the actual world (e.g. the proposition that $q$ iff $q$-in- $w$, where $q$ is false but true in $w$ ). If so, a priori knowability is not even materially factive.

One could respond to the problem by denying that apriority is modally factive. One might reasonably observe that insofar as our main grip on the notion of the apriority of a sentence or a proposition is via the modal notion of apriori knowability, then in the presence of the contingent a priori we should not expect apriority to be modally factive. If one responds in this way to the generalized nesting problem, it is clear that the same response will apply equally to the specific nesting problem for two-dimensionalism. But importantly, the rejection of modal factivity will have been motivated quite independently, so it cannot be seen as an objection to two-dimensionalism per se.

We could simply leave things at that. Still, there remains a sense that we have a grip on a modally factive notion of apriority. To explicate this notion, we cannot understand apriority as a priori knowability. But perhaps another understanding is available.

At this point, there is a happy convergence with the quite different considerations raised by Chalmers in "Actuality and Knowability" (2011b). There, it is argued that if apriority is understood as a priori knowability, then many propositions of the form ' $p$ iff actually $p$ ' are not a priori at all. To maintain the orthodox view that propositions of this sort are always a priori, one needs to understand apriority in nonmodal terms.

On an alternative understanding, we can say that $p$ is a priori iff there is a conclusive a priori warrant for believing $p$. Here a warrant is a sort of idealized propositional justification for a proposition. When there is a proof for $p$, for example, this will yield a conclusive a priori warrant for believing $p$. In some cases (for reasons stemming from "semantic fragility" discussed by Chalmers), it will be impossible to use this warrant to come to know $p$ a priori, 
but $p$ counts as a priori (in the nonmodal sense) all the same.

This nonmodal understanding of apriority offers a potential solution to the generalized nesting problem. One can argue that on the nonmodal understanding, no false proposition is ever a priori, as there is never a conclusive a priori warrant for believing a false proposition. If this is right, then apriority so understood is modally factive.

As a consequence, we must deny (A2) and hold that apriority does not entail necessary apriority. One might think that this is a cost: if there is a conclusive a priori warrant for believing a proposition in one world, such a warrant ought to carry over to every world. However, the two-dimensional framework can provide a natural explanation for why this is not the case.

To see this, we will first spell out a two-dimensional semantics for attitude ascriptions, then extend these to the case of a priori warrant for believing. This semantics will show us why (A2) is false and why apriority is not a pure epistemic operator.

\section{Two-dimensionalism and attitude verbs}

Chalmers $(2002 ; 2011 \mathrm{a})$ develops a two-dimensionalist view according to which attitude verbs are not purely epistemic. One motivation for an impure account stems from examples such as the following.

(4a) Oscar believes that water is a liquid.

(4b) Twin Oscar believes that water is a liquid.

Assume that Oscar is a typical Earth inhabitant, while Twin Oscar is a duplicate of Oscar on Twin Earth (where the watery stuff is XYZ). In this case, the standard intuition is that (4a) is true, while (4b) is false. But in this case, Oscar and Twin Oscar may have beliefs with the same primary intensions (given that primary intensions are a sort of "narrow content"). The moral is that the truth of these ascriptions requires more than a belief with an appropriate primary intension: it also requires an appropriate environment, and in particular an appropriate relation to water.

A similar moral can be drawn for attitude ascriptions involving individuals: 'Oscar believes that Obama is president', for example. To satisfy this ascription, it does not suffice to have a belief with the right primary intension. Oscar must also be related to Obama in the right sort of way.

For a two-dimensionalist, it follows that these attitude ascriptions are not purely epistemic. The obvious moral is that the truth of these ascriptions depends not only on the primary intensions of the subject's beliefs, but also on their secondary intensions.

A natural suggestion is that for (4a) or (4b) to be true, the subject (Oscar or Twin Oscar) must have a belief with the primary intension and the secondary intension associated with 'water is a liquid' in the mouth of the ascriber. The belief Oscar expresses with 'water is a liquid' has the right secondary intension (one true in a world if $\mathrm{H} 2 \mathrm{O}$ is a liquid) as well as the right primary intension, so $(4 \mathrm{a})$ is true. But the corresponding belief that Twin Oscar 
expresses has the wrong secondary intension (one true in a world if XYZ is a liquid), and in fact Twin Oscar has no belief with the right secondary intension, so (4b) is false.

This simplified account is over-simple in a number of respects. The fuller account in Chalmers (2011a) invokes structured intensions (associating complex expressions with complex structures built from the intensions for simple expressions, which themselves consist of ordered pairs of primary intensions and extensions) to allow finer-grained constraints on these ascriptions. It also invokes a coordination relation to allow for variation in primary intensions between ascriber and ascribee. Still, those issues are not central for our current purposes, so we will abstract away from them here and stay with the relatively unstructured account above. One modification to the simple account that is useful for current purposes, however, is to replace the appeal to primary intensions with an appeal to two-dimensional intensions, so that the subject is required to have a belief with the right two-dimensional intension and the right secondary intension. This will still give the right results in the Twin Earth case, as Oscar's and Twin Oscar's beliefs will share the relevant two-dimensional intension while having different secondary intensions.

We can put the resulting account by identifying two-dimensional propositions, the objects of assertion and belief, with ordered pairs consisting of a two-dimensional intension and secondary intension. (These are simplified analogs of the "enriched propositions" in Chalmers 2011a.) We can then say that an attitude ascription is true if and only if the ascribee believes the two-dimensional proposition expressed by the embedded sentence. This yields the following impure semantic clauses for attitude verbs (where ' $B$ ' and ' $K$ ' stand for the believing and knowing relations implicitly relativized to $(v, w))$ :

- $\llbracket \alpha$ believes that $\phi \rrbracket^{v, w}=1$ iff $B\left(\alpha,\left\langle\operatorname{mat}(\phi), \sec _{v}(\phi)\right\rangle\right)$.

- $\llbracket \alpha$ knows that $\phi \rrbracket^{v, w}=1$ iff $K\left(\alpha,\left\langle\operatorname{mat}(\phi), \sec _{v}(\phi)\right\rangle\right)$.

With such a semantics in place, it is easy to see that Forbes' cases pose no problem. Consider his example:

(5) Necessarily, if someone knows that Hesperus has a moon, then Hesperus has a moon.

If 'knows' were a pure epistemic operator, this sentence would yield familiar problems. But on the current impure semantics these problems do not arise. Let ' $H$ ' stand for 'Hesperus has a moon'. Then in worlds where $H$ is false, its secondary intension will be false. It follows that any belief with that secondary intension will be false in that world. If so, the semantics above entails that ' $x$ knows that $H$ ' will be false of all individuals in that world. So (5) is true. ${ }^{7}$ This contrasts with a pure semantics for 'knows' on which the ascribee's primary

\footnotetext{
${ }^{7}$ In terms of the semantic clauses we have that $\llbracket \square\left(\begin{array}{ll}x \text { knows that } H \supset H\end{array}\right) \rrbracket^{v, w}=1$ iff for all worlds $w^{\prime}$ either: (i) $\neg K_{v, w^{\prime}}\left(x,\left\langle\operatorname{mat}(H), \sec _{v}(H)\right\rangle\right)$ or (ii) $\llbracket H \rrbracket^{v, w^{\prime}}=1$. And at any world $w^{\prime}$ where $H$ is false, the knowledge claim will also be false.
} 
intension is all that matters. On this view there could be worlds where $H$ is false but ' $x$ knows that $H^{\prime}$ is true of some individual, so that (5) is false. ${ }^{8}$

Forbes allows that the impure treatment of attitude verbs in Chalmers (2011a) solves his problem (1), but he says that problems still arise for sentences such as (2). He suggests that the framework cannot account for the falsity of the following close relative of (2).

(6) It is epistemically possible that (Hesperus isn't Phosphorus and someone establishes that Hesperus is Phosphorus).

We think this is clearly wrong. Let us take for granted that (6) is true iff the embedded sentence is true at $(v, v)$ for some scenario $v$. This requires that the primary intension of 'Hesperus is Phosphorus' is false at $v$ but that the primary intension of 'Someone establishes that Hesperus is Phosphorus' is true at $v$. On the semantics for attitude ascriptions given above this cannot happen. The ascription 'Someone establishes that Hesperus is Phosphorus' is true at $(v, v)$ if and only if someone in $v$ stands in the establishing relation to the proposition $\left\langle\right.$ mat('Hesperus is Phosphorus'), $\sec _{v}$ ('Hesperus is Phosphorus') $\rangle$. And since someone can't stand in the establishing relation to a false proposition, it follows that $\sec _{v}$ ('Hesperus is Phosphorus') $(v)=1$, i.e. 'Hesperus is Phosphorus' is true at $(v, v)$.

Why is Forbes misled here? He says:

It is the epistemic senses of the conjuncts that matter to the truth-value of [(6)], and the second conjunct, according to Chalmers's views about attitude verbs, is made true at a world $w$ by the subjunctive sense of 'Hesperus is Phosphorus' being established at $w$ in a way that involves observations of Venus. So the epistemic sense of the second conjunct should articulate a Venus-focussed process of establishing a proposition in a scenario, and it is far from obvious why there are no such scenarios that also verify the first conjunct. (Forbes 2012, p. 6)

Forbes' key premise here seems to be that for 'Someone establishes that Hesperus is Phosphorus' to be true at a scenario $v$ on the impure two-dimensionalist account, someone need only establish the secondary intension of 'Hesperus is Phosphorus' at $v$, and that this could be done even in a scenario where the primary intension of 'Hesperus is Phosphorus' is false. (Consider a world in which the morning star, the evening star, and Venus are all distinct, and in which someone establishes that Venus is Venus.) If so, (6) will be false.

However, the key premise is false. Attitude ascriptions require ascribees to have attitudes with the right sort of two-dimensional intension, not just the right sort of secondary intension. Furthermore, epistemic operators can "shift" the secondary intension associated with a given two-dimensional intension. In a scenario where the primary intension of 'Hesperus is not Phosphorus' is true (perhaps Hesperus picks out object $a$ while 'Phosphorus' picks out object $b$ and $a \neq b$ ), the primary intension of 'Someone establishes that Hesperus is not Phosphorus' may be true even though no-one has an item of knowledge with the secondary intension that

\footnotetext{
8In response to Forbes' critique of their view, Gluer and Pagin (2012) accept a sort of pure semantics on which (5) is false. We think that there is no need to bite this bullet.
} 
Venus is not Venus. Rather, what matters is that they have an item of knowledge with the secondary intension picked out by the two-dimensional intension of 'Hesperus is not Phosphorus' in their scenario (a secondary intension true in all worlds where $a \neq b$ ).

This is to say that when scenario shifting operators are involved information about alternative rows of the matrix are sometimes relevant. By shifting the scenario one in a sense can "shift" the secondary intension of an expression - this is just to say that alternative secondary intensions are sometimes relevant in the semantic computation of mixed environments. This is why there is a subscripted scenario parameter on "sec " in the semantic clauses. So the problem Forbes finds here stems not from two-dimensionalism per se, but only from an implausible form of two-dimensionalism, one where the semantics does not have access to the full two-dimensional intension but only to the primary and secondary intensions. ${ }^{9}$

\section{The semantics of apriority}

It remains to give an account of the semantics of 'It is a priori that'. The impurity of ordinary belief and knowledge ascriptions suggests that we should expect an impure account of apriority ascriptions, especially given the internal connections between the notions of belief, knowledge, and apriority.

If we understood 'It is a priori that' as 'It is knowable a priori that', then we could straightforwardly derive the semantics of apriority ascriptions from the semantics of (a priori) knowledge ascriptions and modal claims. However, this understanding of ' $\mathbf{\square}$ ' would be neither modally factive nor a pure epistemic operator. It would not be a pure epistemic operator for reasons discussed in "Actuality and Knowability": for some (actually unknown) sentences $\phi$ of the form ' $\psi$ iff actually $\psi$ ', $\phi$ has a necessary primary intension but $\boldsymbol{\square} \phi$ will be false. It would not be modally factive for reasons discussed under the generalized nesting problem: for some (actually known) sentences $\phi$ of the form ' $\psi$ iff actually $\psi$ ', there will be worlds $w$ at which $\phi$ is true and $\phi$ is false.

For a modally factive understanding of apriority, it is best to pursue the nonmodal understanding from section 3. Here we can say that a proposition $p$ is a priori when there is a conclusive a priori justification for someone to believe it. To assess modal factivity, the key case involves the proposition $q$ actually expressed by a contingent a priori sentence such as 'Julius invented the zip'. The question then is: at a world $w$ where $q$ is false, is there a conclusive a priori justification for believing $q$ ?

A short answer would say "no" on the grounds that there can never be a conclusive propositional justification for believing a false proposition. This may be correct so far as it goes, but there is something deeper going on here.

In a case such as this one, the two-dimensional proposition $q$ cannot even be entertained or asserted in $w$. The primary and secondary intension associated with a mental state or

\footnotetext{
${ }^{9}$ Forbes' construal of the two-dimensionalism apparatus would also have a problem with modals embedded under epistemic operators, such as $\mathbf{\square}(\square \phi \supset \phi)$, and would validate absurdities such as $\square \phi \supset \square \square \phi$. The current semantics handles these straightforwardly.
} 
an utterance always have the same truth-value at the world of utterance: $F(w, w)$, where $F$ and $w$ are the two-dimensional intension and the world of the utterance respectively. But $q$ has a primary intension that is true at $w$ and a secondary intension that is false at $w$. So $q$ cannot be entertained or asserted at $w$.

In cases like this, the two-dimensional intension $F$ determines different secondary intensions with respect to different worlds. With respect to the actual world @, it determines a secondary intension $s_{1}$ that turns on the properties of William Whitworth. With respect to the counterfactual world $w$, it determines a secondary intension $s_{2}$ that turns on the properties of $X$, the inventor of the zip at $w$. It follows that a complex proposition consisting of $F$ and $s_{1}$ cannot be entertained at $w$, as its constituents are not properly aligned. ${ }^{10}$

More precisely, let us say that a two-dimensional proposition $p=\langle F, s\rangle$ (for a twodimensional intension $F$ and a secondary intension $s$ ) is live at a world $w$ iff $F(w)=s$. That is to say that $p$ is live at world $w$ just in case its two-dimensional intension, evaluated at $w$, picks out its secondary intension. Then in cases such as the above, the relevant two-dimensional proposition will not be live at the relevant counterfactual world.

When a two-dimensional proposition $p$ is not live at a world $w$, it is not even available for belief or justification in $w$. As such, there certainly cannot be a propositional justification for believing it in $w$. If so, there cannot be a conclusive a priori propositional justification for believing it. So $p$ is not a priori at $w$, in the sense characterized above. If, however, $p$ is live at $w$ and it has a necessary primary intension, it is plausible that there will be a conclusive a priori propositional justification for believing it. ${ }^{11}$

All this motivates the following two-dimensional semantics for the apriority operator:

- $\llbracket \boldsymbol{\square} \phi \rrbracket^{v, w}=1$ iff

- (i) $\sec _{w}(\phi)=\sec _{v}(\phi)$ [i.e. the liveness constraint]

- (ii) for all $v^{\prime} \llbracket \phi \rrbracket^{v^{\prime}, v^{\prime}}=1$ [i.e. necessary primary intension]

The first clause requires that the relevant proposition be live and the second requires that it have a necessary primary intension. When these conditions are met there will be a conclusive a priori propositional justification for believing the proposition, so the apriority claim will be true.

\footnotetext{
${ }^{10}$ All this is even clearer if one invokes structured intensions. Any thought with the actual primary intension of 'Julius' at $w$ will pick out $X$ rather than William Whitworth, so this thought will not involve the actual secondary intension of 'Julius'. So a complex intension involving the actual primary and secondary intensions of 'Julius' cannot be entertained at $w$. A fully adequate treatment of the apriority operator requires liveness and apriority to be defined for structured intensions along lines discussed in Chalmers (2011a, footnote 24), but we give an unstructured account here for simplicity. The structured account also avoids some odd consequences of the unstructured account that follows (including false instances of $\square(\boldsymbol{\square}(\phi \& \psi) \supset(\mathbf{\square} \& \boldsymbol{\square}))$ when $\phi$ and $\psi$ are not live but $\phi \& \psi$ is $)$. [acknowledgment]

${ }^{11}$ As before, the connection between this sort of apriority and necessity of primary intension may be either substantive or stipulative depending on just how the notions are defined. For example, we can say that $p$ is epistemically possible if $p$ is live and there is no conclusive a priori justification for disbelieving it. We can define scenarios as maximal states that are a priori in this sense. On this understanding it is straightforward that if $p$ is live, $p$ is a priori iff it is true at all scenarios.
} 
For example, consider:

(7) It is a priori that Julius invented the zip.

If the primary intension of 'Julius' works as previous outlined, then clause (ii) above will be satisfied for all $(v, w)$. At (@, @), where @ is the actual world, clause (i) will be satisfied, the two-dimensional proposition will be live, and (7) will be true. The same goes for (@, w) for any world $w$ in which the actual inventor (William C. Whitworth) invented the zip. But for a world $w$ at which someone else invented the zip, clause (i) will be false at (@, $w$ ), the two-dimensional proposition will not be live, and (7) will be false. ${ }^{12}$

When apriority is understood this way, it is modally factive. A contingent a priori sentence $\phi$ (such as 'Julius invented the zip') will have a necessary primary intension and a contingent secondary intension. At a world (such as a world in which actual inventor did not invent the zip) at which the secondary intension of $\phi$ is false, the proposition it expresses will not be live, so the secondary intension of $\phi$ will be false. So $\phi \supset \phi$ will be true in all worlds.

There are some oddities about this notion of apriority. If we said that $p$ is epistemically possible when $p$ is not a priori, then there will be propositions that are epistemically possible even though they are false at all scenarios. So it is best to say (as in footnote 11) that $p$ is epistemically possible when $\neg p$ is not a priori and $p$ is live. A consequence is that apriority and epistemic possibility (or ideal conceivability) are not perfect duals: at a world where $p$ is not live, $\boldsymbol{\square}$ and $\neg p$ are both false. That is, the following modal principle does not hold: $\square(\boldsymbol{\square} \phi \vee \neg \phi)$. Still, this failure of duality does not seem to have any terrible consequences.

We are left with three versions of the apriority operator: one (the operator from section 2) that is purely epistemic but not modally factive, one (the operator from this section) that is modally factive but not purely epistemic, and one (the operator defined in terms of possible a priori knowledge) that is neither. The second of these perhaps comes closest to standard judgments about what counts as a priori, and provides a way to preserve Forbes' intuition that apriority should be modally factive.

If there are any doubts about this modally factive notion of apriority, it is worth noting that the other two understandings are still available. On these understandings, apriority will not be modally factive. But this does not seem to contradict any clear intuitions about ordinary English or any important claims of two-dimensional semantics. Our clear intuitions of modal factivity concern ordinary English terms such as 'know' and 'establish', and these intuitions are respected by the two-dimensional analysis of attitude ascriptions in the previous section. We conclude that two-dimensionalism is not seriously threatened by Forbes' cases involving the nesting of epistemic and modal operators.

\footnotetext{
${ }^{12}$ Other epistemic operators work differently. For example, 'Fred believes that Julius invented the zip' can be true even at a world in which Julius did not invent the zip and in which the two-dimensional proposition expressed by the embedded sentence is not live. So attitude ascriptions do not require a liveness constraint. These cases can instead be handled by Chalmers' (2011a) co-ordination relation, which allows the primary intension of Fred's belief to differ from that involved in the embedded sentence.
} 


\section{References}

Chalmers, D.J. 2002. The components of content. in D.J. Chalmers, (ed), Philosophy of Mind: Classical and Contemporary Readings, Oxford University Press: 608-633.

Chalmers, D.J. 2006. The foundations of two-dimensional semantics. in M. Garcia-Carpintero and J. Macia, (eds), Two-Dimensional Semantics: Foundations and Applications, Oxford University Press: 55-140.

Chalmers, D.J. 2011a. Propositions and attitude ascriptions: A Fregean account. Nô̂s 45(4): 595-639.

Chalmers, D.J. 2011b. Actuality and knowability. Analysis 71(3): 411-419.

Davies, M. and I.L. Humberstone. 1980. Two notions of necessity. Philosophical Studies 38: $1-30$.

Dever, J. 2007. Low-grade two-dimensionalism. Philosophical Books 48: 1-16.

Evans, G. 1979. Reference and contingency. The Monist 62: 161-189.

Forbes, G. 2011. The problem of factives for sense theories. Analysis 71(4): 654-662.

Fritz, P. forthcoming. A logic for epistemic two-dimensional semantics. Synthese.

Gluer, K. and P. Pagin. 2012. Reply to Forbes. Analysis 72(2): 298-303.

Kaplan, D. 1989. Demonstratives. in J. Almog, J. Perry and H. Wettstein (eds), Themes from Kaplan, Oxford University Press: 481-563.

Richard, M. 1993. Sense, necessity, and belief. Philosophical Studies 69: 243-263.

Soames, S. 2005. Reference and Description: The Case Against Two-Dimensionalism. Princeton University Press.

Soames, S. 2007. Actually. Proceedings of the Aristotelian Society, Supplementary Volume 8: $251-277$.

Soames, S. forthcoming. David Lewis's place in analytic philosophy. In Barry Loewer and Jonathan Schaffer (eds.), David Lewis. Wiley. 